\title{
Características estructurales de los clusters industriales en colombia bajo las teorías del desarrollo
}

\author{
pags $50-56$ \\ Grupo de Investigación: Centro de Investigación y Desarrollo Empresarial CINDE \\ Línea de investigación: Lean Manufacturing \\ Andrés Giovanni Guarín Salinase
}

Recibido: 11 de mayo de 2015

\section{RESUMEN}

Las actividades de manufactura en Colombia están concentradas en un grupo tradicional de sectores, los cuales presentan un bajo nivel de valor agregado y baja capacidad de innovación, donde el principal factor de competencia está dado por precio. Como resultado de lo anterior, la industria colombiana, se enfrenta a factores estructurales, entre los cuales están, la falta de derechos de propiedad, asociado principalmente con la baja propensión a invertir en procesos de innovación, al igual que la falta general de infraestructura y la dependencia de las subvenciones públicas. Los esfuerzos de diversificación industrial tomaron una mayor fuerza a finales de la década de los 90's, cuando el gobierno decidió promover un pequeño grupo de sectores, sin embargo, no se ha establecido como incrementar la productividad de aquellos sectores que fueron dejados atrás por la apertura económica, porque la estrategia en su primera fase no planteo una respuesta dirigida a promover una mayor dinámica industrial selectiva.

La respuesta, llegaría por la influencia de Michael Porter, quien realizo una serie de recomendaciones sobre la necesidad urgente para Colombia, de entrar en mercados sofisticados con productos de mayor valor agregado, en los que las empresas nacionales deben usar mejor la información obtenida de sus procesos de intercambio.

Palabras Clave: Desarrollo Económico, Industrialización en Colombia, Clusters
Aceptado: 20 de octubre de 2015

\begin{abstract}
The Colombia's manufacturing activities are concentrated in traditional manufacturing sectors, all of them with low value added and low innovation capacity. The main factor of competition is based on price rather than value added. As a result, the industry faces structural problems, such as lack of property rights, related with low tendency to investment and innovation, as well as lack of transport infrastructure and dependency of public grants. Colombia's diversification efforts started in the late 1990s, when the government decided to promote a small group of sectors. However, has not been established how increase the productivity levels of industrial sectors, they were left behind by the economic opening, the consequence in the first stage of the economic door-open strategy did not raise the productivity, a response aimed at promoting greater industrial dynamics selectively.

The answer to the question, came due to the influence of Michael Porter, who making a series of recommendations on the urgent need for Colombia, to venture into sophisticated markets with higher valueadded products, where domestic firms should better use the information obtained from the exchange processes.
\end{abstract}

Key Words: Economics Development, Colombia Industrialization, Clusters

\footnotetext{
- Ingeniero Industrial, magister en estudios interdisciplinarios de desarrollo económico. Docente investigador programa Ing. Industrial. Fundación Universidad de América. andres.guarin@investigadores.uamerica.edu.co
} 


\section{INTRODUCCIÓN}

Explicar las causas de la industrialización en Colombia y su impacto en el desarrollo de la sociedad colombiana, pasa necesariamente por un ejercicio de revisión histórica, que como dice Hermes Tovar Pinzón (2011, p.4) “...invita a valorar la importancia del tiempo en la comprensión del presente y ver el presente como una congestión de fenómenos que sólo pueden ser analizados en función del pasado". Después de todo, y siguiendo la línea de pensamiento del profesor Tovar, la estructura actual productiva del país, viene siendo forjada desde el mismo colonialismo español, a partir de la ruptura y anulación de los procesos creativos de las sociedades prehispánicas, que ya en tiempos de la república, se ha "favorecido preservar [estas] mismas prácticas en un intento de modernización, dando saltos mortales hacia formas de crecimiento, que aún todavía están ligados a modelos de desarrollo liberales, propuestos por poderes externos" (Tovar, 2011).

Ahondando más en el planteamiento anterior, se distingue que es a partir de 1890 , cuando las actividades manufactureras del país, empezaron a migrar del ámbito artesanal, hacia procesos incipientes de ingenieria, con cada vez mejores niveles de sofisticación, que dieron cabida a una producción industrial más asentada y aceleraron la maduración de mercados regionales. Ahora bien, este cambio correspondió a una serie de transformaciones complejas del tejido social de la época, donde las relaciones de producción cambiaron al introducirse nuevas fuerzas productivas, derivadas inicialmente del comercio y posteriormente de la trilla de café, que permitió una acumulación de capital suficiente, que fue a parar directamente a la creación de industrias de tipo textil, cervecero, de construcción y de mecanizados, entre otras múltiples (Kalmanovitz, 1994).

Así pues, hasta 1905, estas iniciativas empresariales fueron ampliándose y diversificándose, sin contar con mecanismos formales de protección, $y$ eran fundamentalmente las condiciones imperantes en ese tiempo, caracterizadas por una "alta productividad y escalas suficientes de producción, [donde] la dotación de capital fijo, la edad del acervo de capital $y$ el nivel de los salarios" (Kalmanovitz, 1994), definieron un auge en la etapa inicial de la industrialización de Colombia.

Sin embargo, ya para 1912 el arancel que se cobraba en pesos oro por kilo producido, era muy superior (Lévine, 1914), y existía un recargo diferencial por tipo de producto, del orden del $70 \%$, establecido a partir de 1905, con una intención más de tipo fiscal que proteccionista para atender el profundo déficit de la época (Kalmanovitz, 1994).

De estas circunstancias, nace el hecho de que los gobiernos sucesivos desde 1923 a 1970 , buscaran por fuera de las fronteras nacionales a expertos extranjeros, ${ }^{1}$ con el objetivo implantar las mejores prácticas para establecer un equilibrio y crecimiento económico. En consecuencia, el país entra en una tendencia de estructuración y formalización de instituciones, para brindar la confianza suficiente a los inversionistas extranjeros, con el ánimo de facilitar las condiciones para la creación de las primeras empresas con matices similares a las de países desarrollados (Rojas, 2007). Sin embargo, los estudios planteaban que existía una fuerte restricción en la baja productividad de los aparatos industriales nacionales, que estaba ocasionando una limitada asignación de recursos y en extensión, un precario nivel de la calidad de vida de la población.

Con estas condiciones diagnosticadas, se sugirieron dos medidas estructurales, la primera, buscaba la reubicación de la fuerza de trabajo en los centros urbanos, para de esta manera ajustar los salarios a las condiciones de eficiencia industrial (Arevalo, 1997); y la segunda medida, era sustituir la importación de bienes, para que con los excedentes de ahorro, se incrementara la inversión de capital.

1 Dos misiones Kemmerer (1923 y 1930), posteriormente la misión Currie (1949 a 1953), la misión Lebret (1958) y finalmente la misión del Banco Mundial (1970). 
Esta última medida, se convirtió en la estrategia líder y la favorita durante las décadas subsiguientes, que en una primera fase (años cincuentas) ${ }^{2}$ se orientó hacia la consolidación de la producción de sustitución temprana, utilizando como herramientas de protección, niveles arancelarios elevados (Garay, 1998); en la segunda fase, se dio la aparición y posterior consolidación de industrias de bienes intermedios (años sesentas) ${ }^{3}$.

De este modo, para principios de los años setenta, la diversificación industrial se orientó hacia los bienes de sustitución tardia, mostrando resultados satisfactorios, con tasas de crecimiento del $7.0 \%$ anual promedio. Sin embargo, para 1975 los primeros indicadores de deterioro hicieron presencia, asociados a factores como el incremento de los precios del café y a la crisis mundial del petróleo, lo que ocasionó el rompimiento del vínculo entre las fluctuaciones del sector externo y el dinamismo de la acumulación de capital (Chica, 1994; citado en Garay, 1998).

Esto llevó, en los primeros años de la década del ochenta, a presentarse un desequilibrio de las finanzas públicas, derivado de los rezagos cambiarios relacionados con la bonanza cafetera de 1978 y por una caída en los términos de intercambio del país (Garay, 1998), que deterioró el crecimiento de la actividad real.

Dentro de este contexto, ya al final de los años ochenta, y para contrarrestar los efectos de la marcada desaceleración económica, se inicia un periodo de liberación, mediante la reducción de la protección arancelaria, la desregularización de las licencias de importación y la rebaja de los incentivos a las exportaciones, que ocasiono el debilitamiento de sectores industria-

2 Entre 1945 y 1950 se presentó un proceso de modernización industrial con un dinámico crecimiento de la industria manufacturera (al $7.5 \%$ promedio anual), casi el doble del PIB (Garay, 1998).

3 Entre 1959 y 1967 se registró un crecimiento del $6.4 \%$, fortaleciendo la estructura industrial y el empleo (Betry y Thoumi, 1977, citado en Garay, 1998) les que apenas comenzaban a consolidarse en el mercado doméstico y frenó su dinámica de crecimiento.

Lo anterior llevó, a que en 1989, se entrara en un proceso gradual de apertura, donde los elementos del nuevo modelo de desarrollo sostenían que una economía cerrada, "no generaba la dinámica requerida para motivar cambios importantes en su estructura, y que tampoco alentaba la utilización más intensiva de sus recursos para alcanzar mayores niveles de productividad" (Garay, 1998).

Así las cosas, para 1991, se asumió definitivamente el esquema de apertura, motivado en gran parte por la nueva Constitución. Sin embargo, el pacto social conformado entonces, no dejo establecido la manera concreta de aumentar los niveles de productividad de los sectores industriales que quedaron rezagados, debido a que "la estrategia de apertura en su primera fase no planteó una respuesta dirigida a promover una [mayor] dinámica industrial en forma selectiva" (Garay, 1998).

Es así que, la repuesta al interrogante que quedo planteado, vino otra vez de afuera, debido a la influencia de Michel Porter, quien en gran medida rescató los postulados realizados por Alfred Marshall un siglo antes y los matizó con un modelo teórico de cuatro factores, en el que está presente un concepto que autodefine la nueva realidad de los tiempos actuales, el cual fue llamado diamante de competitividad.

Por ello, para 1992 y 1993, el gobierno nacional, nuevamente contrata los servicios de un experto extranjero, esta vez encarnado en Porter, quien dirigió el Proyecto Monitor, realizando una serie de recomendaciones sobre la imperiosa necesidad de Colombia de incursionar en mercados sofisticados con productos de mayor valor agregado, donde las empresas nacionales deberían usar mejor la información obtenida de los procesos de intercambio, ya que "la relación comprador/vendedor en la mayoría de los sectores, se caracterizaba por el antagonismo y por 
la reserva de la información", (Cámara de Comercio de Medellín, 1994).

Bajo este pronóstico, se determinó que la conformación de clusters ${ }^{4}$, era una prioridad crítica para la competitividad de Colombia en el futuro (Cámara de Comercio de Medellín, 1994), donde las empresas ubicadas en un entorno territorial cercano, deberían de manera colaborativa transmitir flujos de innovación a todos los eslabones de la cadena producción, especialmente a los compradores, donde la productividad tendería aumentar, en razón de las complementariedades que comparten los distintos participantes (Rodríguez, 2012).

A consecuencia de lo anterior, y con la transición de cambio siglo, el país se empeñó en sacar adelante los llamados imperativos estratégicos sectoriales, los cuales se han materializado y consolidado en un conjunto de conglomerados, cuyos principales indicadores se han recogido mediante el programa de Apoyo a la Competitividad de Clusters (PACC), establecido mediante un convenio entre el BID y la Universidad de los Andes, quienes en 2012 publicaron un informe que muestra los resultados de un estudio longitudinal de siete años, desde 2004.

Los resultados del estudio referenciado, indican de manera general, que un número importante de los catorce (14) clusters analizados, se encuentran en una etapa incipiente de formación, caracterizada porque cuentan con pocas empresas, no hay cohesión entre los actores y están en proceso de reconocer los benéficos de este modelo de trabajo, debido a un limitado nivel de asociatividad y capacidad de realizar acciones conjuntas (Rodríguez, 2012).

4 Grupo geográficamente denso de empresas e instituciones conexas, pertenecientes a un sector concreto, unidas por rasgos comunes y complementarios entre sí (Porter, 2008).

\section{REFLEXIÓN CRITICA}

Todo el conjunto de elementos expuestos en la parte introductoria de este documento, vistos desde la óptica de las teorías del desarrollo, indican que en el caso de Colombia, no ha sido posible identificar un referente óptimo de modelo de desarrollo que esté acorde en gran medi$\mathrm{da}$, con las tesis argumentadas por los distintos teóricos, dentro de la taxonomía propuesta por Gerald Meiner (2002).

En primer lugar, no se concretaron los cambios gruesos propuestos por las teorías de intervención directa del Estado, donde se debió favorecer y profundizar más en la industrialización, ya que este proceso en el país, se realizó de manera incompleta y muy marginal, solo concentrado en pocos sectores, donde la sustitución de importaciones, como estrategia de fortalecimiento a las empresas manufactureras nacientes, se inició de forma tardía y su duración fue relativamente muy corta, tanto así, que no logró constituir aglomeraciones significativas derivadas de actividades colaborativas de los distintos actores.

Como consecuencia de esto, Colombia, siendo fiel a su herencia colonial, mantuvo la prioridad hacia los mercados extranjeros, haciendo mayor relevancia en aspectos relacionados con las ventajas comparativas que suponían la amplia diversidad natural del territorio, sin llegar a incorporar durante la génesis industrial, elementos de análisis derivados de los planteamientos de los llamados precursores del desarrollo económico (Martinussen, 1997).

Es así como, no se consideraron específicamente los postulados de Alfred Marshall, cuya obra (contemporánea a los inicios de cambio en la producción artesanal colombiana hacia esquemas industriales), consideró de vital importancia para la maximización de las utilidades de las empresas, una localización optima de las mismas, donde los factores de producción definirían las aglomeraciones alrededor de mercados locales (Martinussen, 1997). 
En segundo término, ya bajo el enfoque de las teorías estructuralistas, no se logró interiorizar de manera adecuada, las dinámicas de integración nacional, que facilitarían la configuración de lo que Fracois Perroux llamó "polos de desarrollo"; al no incentivar de manera más decidida la conformación de iniciativas empresariales de alto impacto, que demandarían la aplicación de innovaciones sucesivas, que estuvieran por fuera de la órbita del sector agrícola.

En definitiva, la falta de incorporación de los postulados arriba descritos, en la etapa temprana de la industrialización, acarreó que muchas áreas del territorio colombiano se desligaran unas de otras, debido a la fuerte presencia de una endogamia cultural, resultado de un determinismo geográfico, que definió las maneras y los flujos de interrelación entre las diversas regiones (Yunis, 2003).

Otro elemento fundamental, que no se consideró de forma profunda, ya en épocas de las primeras misiones económicas, fue lo establecido por Joseph Schumpeter, quien sostenía que el desarrollo se alcanzaría en gran medida por medio de las innovaciones técnicas introducidas en la producción, donde los factores utilizados de "nuevas maneras", eran el resultado de la creatividad aportada por las personas (Martinussen, 1997).

La falta de incorporación de los postulados descritos, trajo consigo, que después de 121 años de trasegar económico, se vuelva a mirar con mayor atención estos elementos, pero con la diferencia, que esta vez están siendo regulados por las dinámicas de la globalización y están siendo amparados bajo el concepto de competitividad; el cual, según el Foro Económico Mundial (WEF por su sigla en inglés) $)^{5}$ es considerado como "el conjunto de instituciones, políticas, y factores que determinan la productividad de un país" (Veiga, 2013).

5 Considerado para este trabajo como un referente mundial (no el único), dado su gran peso específico e impacto global.
Es en este sentido, que Colombia no ha logrado configurar un modelo eficiente de cara a lo que realmente significa una competitividad de clase mundial. Por ejemplo, la visión 2032, sugerida por el Consejo Privado de Competitividad en 2007, vista bajo los indicadores recientes del Reporte Global de Competitividad 2014-2015 (Schwab \& Sala-i-Martín, 2014), dan muestras de no llegar a alcanzar mejores niveles, o por lo menos de encontrase en un estado de "letargo", evidenciándose una continuidad en el esquema de crecimiento económico, basado principalmente en la estrategia de venta de commodities.

\section{RECOMENDACIONES Y CONCLUSIONES}

En consideración con todo lo anterior, la hipótesis que subyace en las ideas expuestas, dan cuenta, que antes de estimular la conformación de clusters, es necesario trabajar en una reconfiguración de los aparatos productivos de las empresas manufactureras, que les permita orientar sus decisiones hacia un compromiso más directo con la innovación, analizando el estado de variables criticas relacionadas con la función de producción, al igual que su cultura y el impacto que esta tiene en las personas (Garzón, 2005).

Este nuevo comportamiento organizacional frente a la innovación, pasarla necesariamente por la identificación de un conjunto de habilidades en las personas, que en suma, definirian la construcción de conocimiento y lograrían constituir un ciclo iterativo de mejoramiento continuo ${ }^{6}$, que alcanzaría su materialización en los procesos de fabricación.

Por lo tanto, se debe emprender un proceso de cambio estructural, que redefina la manera como se manufacturan las cosas, ya que los crecimientos sostenidos en productividad dependen en gran medida de un constante cambio en la fiso-

6 El Sistema de Producción de Toyota (TPS), sería un modelo a considerarse 
LÍNEA DE INVESTIGACIÓN: LEAN MANUFACTURING

nomía de los aparatos productivos, para lo cual se necesita una inversión planificada y orientada, que deba ir armonizada con una reducción de los costos para producir productos con mayores niveles de conocimiento.

El rumbo que debe tomar este cambio estructural, pasa por democratizar la economía de mercado, mediante una participación directa de los individuos en la construcción de un crecimiento económico socialmente incluyente, donde las oportunidades estén presentes para todos. Esto conlleva, a que se redefinida el rol del individuo, principalmente en relación con las estructuras organizacionales que conforman su entorno social (incluyendo los clusters), con las cuales tradicionalmente ha mantenido una relación apartada e indiferente, para llegar a establecer, como dice Roberto Mangabeira Unger (2007), "una cooperación en pos de la innovación [...], que permita todo un ejercicio experimental productivo, que este en la vanguardia de los procesos de desarrollo".

De manera que, la producción industrial sea vista como una innovación permanente, que desarrolle un nuevo estilo de manufactura basado más en el experimentalismo, donde la productividad se mida en función de las capacidades rea- les de rediseño de las actividades productivas, que permita un modo de trabajar, más como un ejercicio continuo de imaginación.

Es decir, constituir un vanguardismo productivo (Unger, 2007), aprovechando los avances tecnológicos imperantes, para que las labores rutinarias de transformación involucren cada vez más y de forma gradual procesos de automatización e intercambio de información, y que las personas dediquen más tiempo a idear.

De todo lo anterior se desprende, que el vínculo directo entre la innovación y el progreso económico es del máximo interés por parte de las sociedades. Es a través de la innovación, que se crea y difunde un nuevo conocimiento, lo que aumenta el potencial de la economía para desarrollar nuevos productos y nuevos métodos de funcionamiento más productivos. Por lo tanto, este accionar humano, incide de manera directa en el crecimiento de las naciones y en la manera de interrelacionarnos como especie.

Esto conduce a plantear, que es la empresa, como unidad de cambio social, la que está llamada a liderar los procesos arriba descritos; es la industria quien por definición debe jalonar y motivar el cambio hacia mejores condiciones de vida.

\section{REFERENCIAS}

Arévalo, H. (1997). Misiones Económicas Internacionales en Colombia 1930-1960. Historia Crítica, Vol. 14, p. 7-24

Cámara de Comercio de Medellín para Antioquia. (1994). Creando la ventaja competitiva de Colombia: Informe monitor: resumen ejecutivo. Medellín: Camara de Comercio de Medellin para Antioquia.

Consejo Privado de Competitividad (2007).Informe
Nacional de Competitividad. (Dirección de Estudios e Investigaciones). Bogotá D.C.

Garay, S. L. J., \& Programa de Estudio La Industria de América Latina ante la Globalización Económica. (1998). Colombia, estructura industrial e internacionalización, 1967-1996. Santafé de Bogotá, Colombia: Departamento $\mathrm{Na}$ cional de Planeación.

Garzón Castrillón, M. A. (2005). El modelo intraemprendedor para la innovación.
Bogotá: Centro Editorial Rosarista, Universidad del Rosario.

Kalmanovitz, S. (1994). Economía y Nación: Una breve historia de Colombia. Bogotá: Tercer Mundo Editores.

Schwab, K., \& Sala-i-Martín, X. (2014). The Global Competitiveness Report 2014-2015. Geneva, Switzerland: World Economic Forum

Lévine, V. (1914). Colombia: Physical features, natural resources, means of commu- 
nication, manufactures and industrial development, New York: D. Appleton \& Co.Tovar, P. (2011). La sal del desarrollo. Vicerrectoría de Investigaciones. Colección Seneca. Universidad de los Andes. Bogotá, Colombia.

Martinussen, J. (1997). Society, state, and market: A guide to competing theories of development. London: Zed Books.

Meier, G. M., \& Stiglitz, J. E. (2002). Fronteras de la economía del desarrollo: EI futuro en perspectiva. Bogotá, Colombia: Banco Mundial.
Rodríguez, M. (2012). Gestión de clusters en Colombia: una herramienta para la competitividad. Bogotá: Universidad de los Andes, Facultad de Administración; Banco Interamericano de Desarrollo; Ediciones Uniandes.

Rojas, H.H. (2007). Made in the World is Better: Las Misiones Económicas en Colombia y Nuestro Descreimiento Ancestral. Revista Facultad de Ciencias Económicas, Vol. 15(1), 77-90.

Tovar, P. H. (2011). La sal del desarrollo. Bogotá: Universidad de los Andes, Vicerrectoría de Investigaciones.
Unger, R. M. (2007). Free trade reimagined: the world division of labor and the method of economics. Princeton: Princeton University Press.

Veiga, L. (2013). El Índice de Competitividad Global del Foro Económico Mundial. Revista de Negocios del IEEM, Universidad de Montevideo Vol. 68(6), 80-81.

Yunis, T. E. (2003). Porque somos asi?: Qué pasó en Colombia?: análisis del mestizaje. Bogotá: Editorial Temis. 